\title{
Validación del contenido de un cuestionario para evaluar los hábitos y las prácticas de salud bucodental en los escolares
}

\author{
Adrián Moliner García \\ al339194@uji.es \\ María Belén Moreno Guillamón \\ al261475@uji.es \\ Águeda Cervera Gasch \\ cerveraa@uji.es
}

\section{Resumen}

Introducción: Las dos enfermedades dentales más comunes son la enfermedad periodontal y la caries dental. La infancia se configura como un período esencial para adoptar habilidades y hábitos de vida saludables. Así pues, la educación para la salud constituye un conjunto de acciones de reconocida utilidad en la salud bucodental de los niños. Nuestro objetivo es validar el contenido de un cuestionario para evaluar los hábitos y prácticas de salud bucodental en escolares de $5 .^{\circ}$ y $6 .^{\circ}$ de educación primaria.

Material y método: Se realizó una búsqueda bibliográfica en las principales bases de datos y se elaboró el cuestionario. Para comprobar la validez del contenido, se envió por correo electrónico la versión inicial del cuestionario al panel de expertos, se evaluó la calidad de cada ítem (I-CVI) y la validez global del instrumento (S-CVI). Además, se realizó una prueba piloto con una muestra reducida (10 sujetos) con las mismas características que la muestra objeto de estudio.

Resultados: Se obtuvo un cuestionario formado por tres bloques y una validez global de 0,92 . En el primer bloque, se valoraron los datos sociodemográficos, y, en el segundo, formado por 23 ítems, los hábitos y prácticas de salud bucodental. En el tercero, el índice de O'Leary. Los resultados de la prueba piloto mostraron que el cuestionario era entendido por la población diana.

Conclusiones: El cuestionario presenta unos resultados de validez de contenido adecuados, por lo que es posible utilizarlo para futuras investigaciones.

Palabras clave: validación, contenido, cuestionario, salud bucodental, escolares. 


\section{Abstract}

Introduction: Periodontal disease and dental caries are the two most common dental diseases. Childhood is an essential period to adopt skills and habits of healthy life. So, education for health constitutes a set of important actions in the dental hygiene of children. Our objective is to validate the content of a questionnaire to evaluate oral health habits and practices in 5th and 6th grade primary school students.

Material and method: A bibliographic search was made in the main databases and the questionnaire was elaborated. To validate the content, the initial version of the questionnaire was sent by e-mail to the panel of experts, the quality of each item was evaluated (I-CVI), as well as the overall validity of the instrument (S-CVI). In addition, a pilot test was carried out with a reduced sample (10 subjects) with the same characteristics as the sample under study.

Results: A questionnaire consisting of three blocks and a global validity of 0.92 was obtained. In the first block, the sociodemographic data were valued, and, in the second, consisting of 23 items, oral health habits and practices were valued. In the third, the O'Leary index. The results of the pilot test showed that the questionnaire was understood by the target population.

Conclusion: The questionnaire presented adequate content validity results, so it is possible to use it for future research.

Keywords: validation, contents, questionnaire, dental hygiene, schoolchildren.

\section{Introducción}

Actualmente, las dos enfermedades dentales más comunes que afectan a los escolares son la gingivitis y la caries dental (Cardozo, Bessone, Pérez, Vaculik y Sanz 2017). Además, estas son consideradas un problema de salud pública en el mundo por su alta incidencia e impacto en la salud de la población (Herrera Serna y López Soto 2018).

La infancia constituye un periodo fundamental para adoptar hábitos de vida saludables, dado que estos se mantendrán en la etapa adulta (Generalitat Valenciana 2013). Es esencial la conservación de la salud dental y, actualmente, se enfatiza en la importancia de establecer las atenciones necesarias ya desde el primer año de vida, por medio del trabajo coordinado de todos los profesionales que atienden la salud infantil. El enfoque preventivo y las actividades que se aplican desde los primeros años de vida son claves para el éxito de los programas de salud (Bosch Robaina, Rubio Alonso y García Hoyos 2012). Es de importancia establecer hábitos saludables de alimentación y de higiene dental, vigilar la salud oral para detectar riesgos o lesiones incipientes y visitar al estomatólogo al menos una vez al año (Sosa Rosales 2009).

En cuanto a los problemas de salud bucodental en España, el Instituto Nacional de Estadística (INE) (2018) muestra en la tabla 1 el estado de la dentadura en una muestra de población de 5 a 14 años con independencia de género en España: 
Tabla 1

Estado de la dentadura en ambos sexos y edad de 5 a 14 años (fuente: INE)

\begin{tabular}{ccccc}
\hline $\begin{array}{c}\text { Tiene } \\
\text { caries }\end{array}$ & $\begin{array}{c}\text { Le han } \\
\text { extraído } \\
\text { dientes/muelas }\end{array}$ & $\begin{array}{c}\text { Tiene } \\
\text { dientes/muelas } \\
\text { empastados } \\
\text { (obturados) }\end{array}$ & $\begin{array}{c}\text { Le sangran las } \\
\text { encías al } \\
\text { cepillarse o } \\
\text { espontáneamente }\end{array}$ & $\begin{array}{c}\text { Los } \\
\text { dientes } \\
\text { que tiene } \\
\text { están } \\
\text { sanos }\end{array}$ \\
\hline 571 & 669 & 1374 & 262 & 3746 \\
\hline
\end{tabular}

Según la encuesta de salud oral en España del 2015 (Bravo Pérez et al. 2016), la prevalencia de caries en dentición permanente a los 12 y 15 años es del 33,30\% y del $43,20 \%$ respectivamente. Además de esto, la frecuencia en los hábitos bucodentales saludables en la población infanto-juvenil (de 2 a 15 años) residente en España se aleja de las recomendaciones de las sociedades científicas. Existe una menor frecuentación de los servicios dentales, una menor frecuencia de cepillado dental diario y una alta ingesta de dieta de riesgo cariogénico (Almerich-Silla, Boronat Ferrer, Montiel Company y Iranzo Cortés 2014).

Estos problemas dentales se pueden evitar siguiendo unas medidas de prevención, como las siguientes: medidas de higiene bucodental, uso de fluoruros y medidas dietéticas (Generalitat Valenciana 2013). Además, en una higiene oral efectiva es imprescindible eliminar la placa bacteriana de todas las superficies dentarias, al menos una vez al día (Junta de Andalucía 2012).

Por otro lado, la Organización Mundial de la Salud (OMS) (1983) define la educación para la salud (EpS) como «cualquier combinación de actividades de información y educación que lleve a una situación en que la gente desee estar sana, sepa cómo alcanzar la salud, haga lo que pueda individual y colectivamente para mantener la salud y busque ayuda cuando la necesite». Esta trata de capacitar a las personas para que puedan ejercer su libertad de elegir las pautas de conducta que más les interesen para mejorar su calidad de vida.

Finalmente, el entorno escolar se considera el idóneo para realizar EpS en estas edades ya que los alumnos permanecen en el centro docente gran parte de su tiempo $y$, además, es la edad a la que comienzan a desarrollar hábitos importantes que repercutirán a lo largo de su vida (Fernández Candela, Rebolledo Malpica y Velandia Mora 2006). Esto propicia que los alumnos y alumnas sean agentes activos de salud al tratar de difundir ellos mismos informaciones, actitudes y pautas de conducta (Gavidia Catalán 2001, 505-516).

Sin embargo, en la revisión de la literatura no se encontraron cuestionarios validados que permitan estudiar los hábitos y las prácticas de higiene bucodental en escolares. Es por ello que, tras observar los datos epidemiológicos sobre los problemas de salud bucodental y la importancia de mantener unos hábitos adecuados, se planteó como objetivo principal de este estudio diseñar y validar el contenido de un cuestionario para evaluar los hábitos y prácticas de salud bucodental en escolares de $5 .^{\circ}$ y $6 .^{\circ}$ de educación primaria. 


\section{Material y método}

\section{Participantes}

Para realizar la validación del cuestionario fue necesario un panel de expertos constituido por seis miembros: una profesora asociada de la Unidad Predepartamental de Enfermería de la Universitat Jaume I y enfermera pediátrica, un odontoestomatólogo en consulta de odontología, una higienista bucal en consulta de odontología, una higienista bucal y enfermera, una enfermera a cargo del Programa de Salud Bucodental y una doctorada en odontología. Estos eran expertos en la materia y, además, contaban con más de diez años de experiencia.

Por otro lado, se realizó una prueba piloto y pretest cognitivo a una muestra con características fundamentales semejantes a la población diana de estudio (Casas Anguita, Repullo Labrador y Donado Campos 2003; Arribas 2004, 23-29; Amezcua 2015), con un grupo de 10 niños/as entre $5 .^{\circ}$ y $6 .^{\circ}$ de educación primaria de forma aleatorizada, pertenecientes al colegio Puértolas Pardo de Alcora (Castellón).

\section{Instrumento}

Para el diseño del instrumento, se realizó una búsqueda bibliográfica donde se encontró un estudio sobre "Conocimientos sobre salud bucodental y evaluación de higiene oral antes y después de una intervención educativa en niños de 9-10 años» de Bosch Robaina, Rubio Alonso y García Hoyos (2012) y otro estudio sobre una «Encuesta sobre hábitos higiénicos orales en la población adolescente de la Comunidad Valenciana (2004)» de Almerich y Montiel (2006) a partir de los cuales se redactaron los ítems, obteniendo la versión inicial del cuestionario. Después se realizó una validación del contenido y una prueba piloto, llegando a obtener la versión final del cuestionario formada por 29 ítems separados en 3 bloques.

\section{Procedimiento de desarrollo y validación del instrumento}

El cuestionario para evaluar los hábitos y las prácticas de salud bucodental en los escolares se desarolló a través de las pautas propuestas por Casas Anguita, Repullo Labrador y Donado Campos (2003). En primer lugar, los investigadores definieron el constructo que se deseaba medir (los hábitos y las prácticas de salud bucodental en los escolares). Posteriormente, en la fase de construcción del cuestionario se realizó una revisión bibliográfica utilizando los descriptores: Oral health, Child, Dental Care for Children y Oral Hygiene Index, en las bases de datos PubMed, Biblioteca Cochrane y LILACS, con ayuda de los operadores booleanos $A N D$ y OR. Se aplicó el filtro de temporalidad inferior a 5 años así como el acceso a texto completo.

Sin embargo, debido a los pocos resultados obtenidos en las principales bases de datos y al ver que ningún artículo contenía un cuestionario validado útil para el estudio, se realizó una búsqueda complementaria en la biblioteca virtual SciELO y en el metabuscador Google Scholar con el fin de obtener variables que pudieran estar relacionados con el objetivo del cuestionario. Esta revisión estableció la primera versión de los ítems del cuestionario. Así, la redacción de los 19 ítems se basó en los trabajos de Bosch Robaina, Rubio Alonso y García Hoyos (2012) y Almerich y Montiel (2006) y se organización en 3 dimensiones: variables sociodemográficas, variables sobre hábitos y prácticas de salud bucodental y variable resultado. 
A continuación, se realizó una validación del contenido para obtener evidencia de que los ítems elegidos son indicadores de lo que se pretende medir (Arribas 2004, 23-29). La validación del contenido se realizó a través del índice de validez de contenido (Index of content validity) y un panel de expertos utilizando la metodología de Polit y Beck (2006).

A cada uno de los expertos se le envió por correo electrónico la versión inicial del cuestionario. Se les solicitó que valorasen, mediante una escala Likert ascendente de cuatro puntos (1=Nada adecuado, 2=Poco adecuado, 3=Adecuado, 4=Muy adecuado), la idoneidad y pertinencia de los ítems que componían cada dimensión (Urrutia Egaña, Barrios Araya, Gutiérrez Núñez y Mayorga Camus 2014; Pedrosa, Suárez-Álvarez y García-Cueto 2014; Matas 2018). Del mismo modo, se les solicitó que plantearan, si creían conveniente, nuevos ítems en un espacio abierto donde podían expresar su opinión general sobre el cuestionario. Los expertos dispusieron de cinco días para completar la validación.

Finalmente, con la segunda y definitiva versión del cuestionario, se realizó la prueba piloto y el pretest cognitivo para evaluar si la aplicación de la encuesta era factible en el momento y grupo seleccionado y si los ítems respondían a los objetivos del cuestionario (Casas Anguita, Repullo Labrador y Donado Campos 2003). El cuestionario que se llevó a cabo en la prueba piloto estaba compuesto por 29 ítems. Para evaluar la prueba piloto y el pretest cognitivo el alumnado realizó el cuestionario al mismo tiempo, pregunta por pregunta, en voz alta, para resolver cualquier tipo de duda existente y poder valorar la comprensión de los ítems y el tiempo de cumplimentación.

\section{Análisis estadísticos}

Se calculó el índice de validez de contenido (validez adecuada I-CVI>0,75) (Vargas Salgado, Máynez-Guaderrama, Cavazos Arroyo y Cervantes Benavides 2016) para cada ítem, así como el coeficiente de validez global (validez adecuada I-CVI>0,8) (Polit, Beck y Owen 2007). Estos cálculos se realizaron para el cuestionario y en cada uno de los bloques. Los resultados de la validez de contenido y las consideraciones de los expertos se tuvieron en cuenta en la elaboración de la segunda versión del cuestionario.

\section{Resultados}

Las fases que se realizaron para el proceso de validación del contenido del cuestionario se resumen en la siguiente figura:

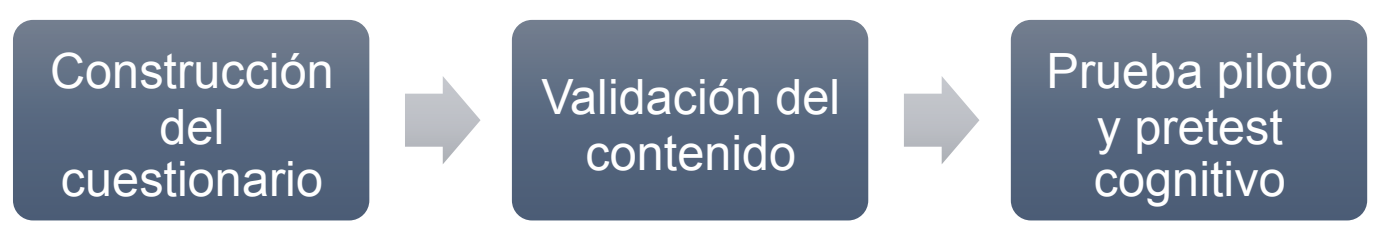

Figura 1. Fases del proceso de validación del contenido del cuestionario (fuente: elaboración propia). 
Al principio, se encontraron dos artículos que sirvieron para la construcción del instrumento, obteniendo un cuestionario formado por tres bloques. La siguiente tabla muestra las modificaciones que se han ido realizando al cuestionario a lo largo del proceso de validación del contenido:

Tabla 2

Número de ítems del cuestionario por fases (fuente: elaboración propia)

\begin{tabular}{cccc}
\hline \multicolumn{4}{c}{ Proceso de validación del contenido por fases } \\
\hline Fase & Actividad & $\begin{array}{c}\text { Número de } \\
\text { ítems }\end{array}$ & $\begin{array}{c}\text { Número de } \\
\text { bloques }\end{array}$ \\
\hline I & Construcción del cuestionario & 19 & 3 \\
II & Validación del contenido del cuestionario & 29 & 3 \\
III & Prueba piloto y pretest cognitivo & 29 & 3 \\
\hline
\end{tabular}

Tras la búsqueda bibliográfica realizada se diseñó un cuestionario compuesto por 3 bloques. Un primer bloque sobre datos sociodemográficos, que incluía el curso escolar, la edad, el género y la ocupación laboral del padre y de la madre. Un segundo bloque sobre datos de hábitos y prácticas de salud bucodental compuesto por 23 ítems con preguntas cerradas, con diferentes opciones de respuesta (tipo test, verdadero y falso), y preguntas abiertas. Por último, un tercer bloque donde se realizaba la evaluación mediante el índice de O'Leary, además de añadir cualquier observación sobre la técnica de cepillado dental del sujeto.

En segundo lugar, tras recibir las distintas respuestas del panel de expertos, se realizó una evaluación de cada uno de los ítems mediante el índice de validez de contenido y se calculó la validez global del instrumento, que se pueden observar en la tabla 3 :

Tabla 3

Índice de validez de contenido (I-CVI) por bloques del cuestionario

(fuente: elaboración propia)

\begin{tabular}{c|c}
\hline Primer bloque & 0.8 \\
Segundo bloque & 0.96 \\
Tercer bloque & 1 \\
Validez global (S-CVI) & 0.92 \\
\hline
\end{tabular}

La puntuación global del S-CVI fue del 0,92 y todos los bloques obtuvieron puntuaciones de $\mathrm{S}-\mathrm{CVI}$ superiores al 0,8 . Además, en la siguiente tabla se muestran los ítems que fueron eliminados de la versión inicial por obtenerse valores de I$\mathrm{CVI}<0,75$ :

Tabla 4

Ítems eliminados de la versión inicial por obtener (fuente: elaboración propia)

\begin{tabular}{cc}
\hline Ítems & I-CVI \\
\hline ¿Cuántos hermanos tienes? & 0,67 \\
¿Tomaste de pecho de tu madre cuando eras pequeño/a? & 0,5 \\
\hline
\end{tabular}


Del mismo modo, se amplió el bloque sociodemográfico y el bloque sobre hábitos y prácticas de salud bucodental con algunos ítems $y$, por otra parte, se realizaron pequeños cambios de redacción. Finalmente, se obtuvo la segunda versión y definitiva del cuestionario compuesto por los mismos bloques que la versión inicial, con un total de 29 ítems.

En relación con la prueba piloto y el pretest cognitivo realizados con 10 sujetos similares a la población objeto de estudio, se observó que la cumplimentación de la segunda versión del cuestionario tuvo una duración de 15 minutos. Todos los sujetos contestaron a las preguntas propuestas por el cuestionario a la misma vez que el resto y en voz alta, con el objetivo de resolver cualquier duda existente. Tras su realización, se obtuvo que algunos de los ítems eran incomprensibles por los sujetos y precisaban una reformulación o explicación. De este modo, se cambió el orden de las preguntas y se aclararon los ítems. Con ello, se obtuvo un cuestionario compuesto por los mismos bloques que el anterior, pero con los ítems reformulados. Finalmente, se obtuvo una versión final del cuestionario (anexo 1).

\section{Discusión y conclusiones}

La validez del contenido del instrumento se decidió realizar a través de un panel de expertos por la facilidad que suponía ponerse en contacto con los mismos, siguiendo el modelo de Fehring (Urrutia Egaña, Barrios Araya, Gutiérrez Núñez y Mayorga Camus 2014). Aunque algunos estudios recomiendan ampliar el número de participantes (Gómez Fernández-Vegue y Menéndez Orenga 2015; Carrion, Soler y Aymerich 2015, 13-22), al parecer, no está establecido un número concreto de participantes (Escobar-Pérez y Cuervo-Martínez 2008, 27-36). Todos los expertos decidieron que los ítems «¿Cuántos hermanos/as tienes?» y «¿Has recibido lactancia materna?» eran irrelevantes en el cuestionario, por lo que se eliminaron de la primera versión. De igual modo, aportaron ítems para evaluar una alimentación saludable, técnica de cepillado dental, cepillado de la lengua, uso de flúor en dentífrico y colutorio y uso de seda dental. Además, se comentó ampliar el cuestionario con un bloque para evaluar la alimentación de la muestra, incentivando una alimentación saludable siguiendo una dieta con bajo riesgo cariogénico, pero se decidió no incluirlo puesto que se prefirió evitar que la cumplimentación del cuestionatio creara cansancio. Sin embargo, todos los expertos estaban de acuerdo con el índice de O'Leary como método para evaluar la técnica de cepillado dental de los sujetos y, también, creyeron que el cuestionario era novedoso, en cuanto el tema, y útil en un futuro, ya que existe una falta de implicación por parte del personal sanitario por la salud de los más pequeños y la enseñanza de su autocuidado.

Finalmente, para dar más validez al instrumento, se realizó una prueba piloto y pretest cognitivo a una muestra de 10 sujetos. Varios estudios recomiendan realizar la prueba con una muestra que oscile entre los 15 y 50 sujetos (Casas Anguita, Repullo Labrador y Donado Campos 2003; Arribas 2004, 23-29). Este método sirvió para valorar que el tiempo de cumplimentación del cuestionario era adecuado para la edad que se dirigía, teniendo en cuenta que en edades menores es difícil mantener la concentración de los individuos. De igual modo, se eliminaron algunas opciones de respuesta de los ítems por incompresión de los individuos (por ejemplo, indiferente). Se reescribieron algunos ítems que se encontraban escritos incorrectamente y se añadieron explicaciones en algunos otros, debido al desconocimiento del tema por la muestra, como el término seda dental. Finalmente, se añadió en algunos ítems que es posible la elección de más de una opción de respuesta, para facilitar más la realización del cuestionario. 
En relación con el objetivo planteado anteriormente, se llega a la conclusión de que ha sido posible la construcción y validación del contenido de un cuestionario que sirvió para evaluar los hábitos y las prácticas de salud bucodental en los escolares de $5 .^{\circ}$ y $6 .^{\circ}$ de educación primaria, por lo que es posible utilizarlo para futuras investigaciones. En cuanto a las futuras líneas de investigación, se visualiza continuar con el proceso de validación del cuestionario, realizando una validación mucho más exhaustiva. En este caso, ampliando el número y la variedad de expertos y, posteriormente, realizando una prueba piloto y pretest cognitivo con una mayor muestra.

\section{Anexos}

Tabla 5

Ítems incluidos en la versión final del cuestionario (fuente: elaboración propia)

\begin{tabular}{|c|c|}
\hline \multicolumn{2}{|c|}{ Bloque I - Variables sociodemográficas } \\
\hline Edad & Curso académico \\
\hline Sexo & Ocupación laboral de padre y madre \\
\hline \multicolumn{2}{|c|}{ Bloque II - Variables sobre hábitos y prácticas de salud bucodental } \\
\hline ¿Cómo aprendiste a cepillarte los dientes? & $\begin{array}{c}\text { ¿Hasta qué edad te revisaban que te } \\
\text { cepillabas bien los dientes? }\end{array}$ \\
\hline $\begin{array}{c}\text { ¿Cuántas veces has visitado el odontólogo o } \\
\text { dentista en el último año? }\end{array}$ & ¿Has tenido caries alguna vez? \\
\hline ¿Cuántas caries que recuerdes? & ¿Cuántas veces al día te cepillas los dientes? \\
\hline ¿Qué técnica utilizas en el cepillado? & ¿Qué tipo de cepillo dental utilizas? \\
\hline $\begin{array}{c}\text { ¿Compartes el cepillo dental con otra } \\
\text { persona? }\end{array}$ & $\begin{array}{c}\text { ¿Usas una pasta de dientes que tenga flúor } \\
\text { para el cepillado dental? }\end{array}$ \\
\hline $\begin{array}{l}\text { ¿Qué cantidad de flúor contiene la pasta } \\
\text { dental de uso habitual? }\end{array}$ & $\begin{array}{l}\text { ¿Qué cantidad de pasta de dientes utilizas en } \\
\text { el cepillo dental? }\end{array}$ \\
\hline ¿Te cepillas la lengua? & $\begin{array}{l}\text { Después de cepillarte los dientes, ¿te } \\
\text { enjuagas la boca con agua? }\end{array}$ \\
\hline $\begin{array}{c}\text { Después de cepillarte los dientes, ¿usas un } \\
\text { enjuague bucal que tenga flúor? }\end{array}$ & $\begin{array}{l}\text { ¿En qué momento del día utilizas un } \\
\text { enjuague bucal que contenga flúor? }\end{array}$ \\
\hline $\begin{array}{c}\text { Después de usar el enjuague bucal, ¿te } \\
\text { enjuagas la boca con agua? }\end{array}$ & ¿Usas seda dental con el cepillado dental? \\
\hline $\begin{array}{l}\text { ¿Utilizas en el cepillado dental algún otro } \\
\text { material que tenga flúor? }\end{array}$ & $\begin{array}{l}\text { ¿Tus padres, hermanos o algún familiar te } \\
\text { siguen cepillando los dientes? }\end{array}$ \\
\hline $\begin{array}{l}\text { ¿Tus padres, hermanos o algún familiar te } \\
\text { repasan los dientes después de cepillártelos? }\end{array}$ & $\begin{array}{l}\text { ¿Cuántas veces al día, de forma habitual, } \\
\text { comes algo dulce, como pasteles, golosinas, } \\
\text { chocolate, azúcar, bollería, etc.? }\end{array}$ \\
\hline $\begin{array}{l}\text { ¿Qué sueles traer normalmente de almuerzo } \\
\text { al colegio? }\end{array}$ & $\begin{array}{l}\text { ¿Te gustaría aprender más sobre cómo } \\
\text { cuidar de tus dientes? }\end{array}$ \\
\hline \multicolumn{2}{|c|}{ Bloque III: Variable resultado } \\
\hline O'Leary y obse & ciones del cepillado dental \\
\hline
\end{tabular}




\section{Referencias bibliográficas}

Almerich-Silla, José Manuel y José María Montiel Company. 2006. «Encuesta sobre hábitos higiénicos orales en la población adolescente de la Comunidad Valenciana». RCOE, 11(2): 195-201. Recuperado de: http://scielo.isciii.es/pdf/rcoe/v11n2/195_201.pdf [Consultado el 4 de junio de 2019].

Almerich-Silla, José Manuel, M. ${ }^{a}$ Teresa Boronat Ferrer, José María Montiel Company y José Enrique Iranzo Cortés. 2014. «Caries prevalence in children from Valencia (Spain) using ICDAS II criteria». Medicina oral, patologia oral y cirugia bucal, 19(6), e574.

Amezcua, Manuel. 2015. «Cómo estructurar un Proyecto de Investigación con prueba piloto». Recuperado de: http://index-f.com/gomeres/?p=996 [Consultado el 4 de junio de 2019].

Arribas, M. ${ }^{a}$ Concepción. 2004. «Diseño y validación de cuestionarios». Matronas profesión, 5(17): 23-29. Recuperado de: https://www.federacionmatronas.org/wp-content/uploads/2018/01/vol5n17pag23-29.pdf [Consultado el 4 de junio de 2019].

Bosch Robaina, Ruslan, Margarita Rubio Alonso y Felisa García Hoyos. 2012. «Conocimientos sobre salud bucodental y evaluación de higiene oral antes y después de una intervención educativa en niños de 9-10 años». Avances en odontoestomatología, 28(1): 17-23. Recuperado de:

http://scielo.isciii.es/pdf/odonto/v28n1/original2.pdf [Consultado el 4 de junio de 2019].

Bravo Pérez, Manuel et al. 2016. «Encuesta de Salud Oral en España 2015». Revista RCOE, 21 (suplemento 1): 8-48. Recuperado de:

https://www.consejodentistas.es/comunicacion/actualidad-del-

consejo/publicaciones-del-consejo/revistas-del-consejo/revista-rcoe/item/1238encuesta-de-salud-oral-en-espana-2015-revista-rcoe-junio-2016-vol-21suplemento-1.html [Consultado el 4 de junio de 2019].

Cardozo, Beatriz Juana, Gabriela Bessone, Silvia Rita Pérez, Patricia Alejandra Vaculik y Elena Griselda Sanz. 2017. «Evaluación del estado de Salud Bucal de niños en 4 y 5 años que asisten al Centro de Desarrollo Infantil Mitai Roga N. ${ }^{\circ}$ V, de la ciudad de Corrientes Capital». Revista Digital, 1(1). Recuperado de: http://revistas.unne.edu.ar/index.php/rdo/article/view/2939/2616 [Consultado el 4 de junio de 2019].

Carrion, Camen, Mònica Soler y Marta Aymerich. 2015. «Análisis de la validez de contenido de un cuestionario de evaluación del aprendizaje basado en problemas: un enfoque cualitativo». Formación universitaria, 8(1): 13-22. Recuperado de:

https://scielo.conicyt.cl/scielo.php?script=sci_arttext\&pid=S0718-

$50062015000100003 \&$ Ing=en\&nrm=iso\&tlng=en [Consultado el 4 de junio de 2019].

Casas Anguita, Juana, José Ramón Repullo Labrador y Juan de Mata Donado Campos. 2003. "La encuesta como técnica de investigación. Elaboración de cuestionarios y tratamiento estadístico de los datos (I)». Atención primaria, 31(8): 527-538. Recuperado de:

http://www.unidaddocentemfyclaspalmas.org.es/resources/9+Aten+Primaria+20 03.+La+Encuesta+I.+Custionario+y+Estadistica.pdf [Consultado el 4 de junio de 2019].

Escobar-Pérez, Jazmine y Ángela Cuervo-Martínez. 2008. «Validez de contenido y juicio de expertos: una aproximación a su utilización». Avances en medición, 6(1): 27-36. Recuperado de:

http://www.humanas.unal.edu.co/psicometria/files/7113/8574/5708/Articulo3_Ju icio_de_expertos_27-36.pdf [Consultado el 4 de junio de 2019]. 
Fernández Candela, Fernando, Dinoria Margarita Rebolledo Malpica y Ana Luisa Velandia Mora. 2006. «Salud escolar, ¿por qué el profesional de enfermería en las escuelas españolas?». Revista Hacia la promoción de la salud, 11. Recuperado de: http://www.redalyc.org/articulo.oa?id=309126325004 [Consultado el 4 de junio de 2019].

Gavidia Catalán, Valentín. 2001. "La transversalidad y la escuela promotora de salud». Revista española de salud pública, 75(6): 505-516. Recuperado de: http://scielo.isciii.es/scielo.php?script=sci_arttext\&pid=S113557272001000600003 [Consultado el 4 de junio de 2019].

Generalitat Valenciana, Conselleria de Sanitat. 2013. Programa de salud bucodental infantil en la Comunitat Valenciana. Valencia. Recuperado de: http://www.ceice.gva.es/documents/162783553/162784602/manual_PSB_2013. pdf/408fea55-ab6a-4ef9-9b50-553b33d5e99f [Consultado el 4 de junio de 2019].

Gómez Fernández-Vegue, Marta y Miguel Menéndez Orenga. 2015. «Validación de un cuestionario sobre conocimientos y habilidades en lactancia materna». Anales de Pediatría, 83 (6): 387-396. doi: 10.1016/j.anpedi.2015.01.006

Herrera Serna, Brenda Yuliana y Olga Patricia López Soto. 2018. «Evaluación a 72 meses de una estrategia de prevención en salud oral en escolares». Revista Española de Salud Pública, 92. Recuperado de:

http://www.mscbs.gob.es/biblioPublic/publicaciones/recursos_propios/resp/revis ta_cdrom/VOL92/ORIGINALES/RS92C_201808061.pdf

Instituto Nacional de Estadística. 2018. Estado de la dentadura según sexo y grupo de edad. Población de 0 a 14 años. Recuperado de:

https://www.ine.es/jaxi/Datos.htm?path=/t15/p419/a2017/p02/I0/\&file=02031.px [Consultado el 4 de junio de 2019].

Junta de Andalucía, Consejería de Salud. 2012. Programa de promoción de la salud bucodental + propuesta didáctica. Sevilla: Junta de Andalucía, Consejería de Igualdad, Salud y Políticas Sociales.

Matas, Antonio. 2018. "Diseño del formato de escalas tipo Likert: un estado de la cuestión». Revista electrónica de investigación educativa, 20(1): 38-47. Recuperado de: http://www.scielo.org.mx/pdf/redie/v20n1/1607-4041-redie-2001-38.pdf

Pedrosa, Ignacio, Javier Suárez-Álvarez y Eduardo García-Cueto. 2014. «Evidencias sobre la validez de contenido: avances teóricos y métodos para su estimación». Acción psicológica, 10(2): 3-18. Recuperado de: http://scielo.isciii.es/pdf/acp/v10n2/02monografico2.pdf [Consultado el 4 de junio de 2019].

Polit, Denise F. y Cheryl Tatano Beck. 2006. «The content validity index: are you sure you know what's being reported? Critique and recommendations». Research in nursing \& health, 29(5): 489-497.

Polit, Denise F., Cheryl Tatano Beck y Steven V. Owen. 2007. «ls the CVI an acceptable indicator of content validity? Appraisal and recommendations». Research in nursing \& health, 30(4): 459-467.

Sosa Rosales, Maritza de la Caridad. 2009. Promoción de salud bucodental y prevención de enfermedades. Recuperado de: http://www.sld.cu/galerias/pdf/uvs/saludbucal/promprev.pdf [Consultado el 4 de junio de 2019].

Urrutia Egaña, Marcela, Silvia Barrios Araya, Marina Gutiérrez Núñez y Magdalena Mayorga Camus. 2014. «Métodos óptimos para determinar validez de contenido». Educación Médica Superior, 28(3): 547-558. Recuperado de: http://scielo.sld.cu/scielo.php?script=sci_arttext\&pid=S086421412014000300014 [Consultado el 4 de junio de 2019].

Vargas Salgado, Marisa, Aurora Irma Máynez-Guaderrama, Judith Cavazos Arroyo y Laura Elizabeth Cervantes Benavides. 2016. "Validez de contenido de un 
instrumento de medicion para medir el liderazgo transformacional (Content Validity of a Measuring Instrument for Transformational Leadership)». Revista Global de Negocios, 4(1): 35-45. Recuperado de:

https://papers.ssrn.com/sol3/papers.cfm?abstract_id=2659369 [Consultado el 4 de junio de 2019]. 